\title{
Atuação do enfermeiro na prevenção e detecção do câncer do colo uterino para a
}

\section{melhora de vida de mulheres}

\author{
Nurse's role in the prevention and detection of cervical cancer to improve women's lives \\ El papel de la enfermera en la prevención y detección del cáncer de cuello uterino para mejorar la
}

vida de las mujeres

Recebido: 30/05/2021 | Revisado: 06/06/2021 | Aceito: 08/06/2021 | Publicado: 22/06/2021
Liane Bahú Machado
ORCID: https://orcid.org/0000-0002-1356-373X
Universidade Federal de Santa Maria, Brasil
E-mail: lianemachado61 @gmail.com
Silvana Carloto Andres
ORCID: https://orcid.org/0000-0001-6726-7947
Universidade Federal de Santa Maria, Brasil
E-mail: silvana.andres@ yahoo.com.br
Marjana Pivoto Reginaldo
ORCID: https://orcid.org/0000-0002-2260-8207
E-mail: marjanapivotto@gmail.com
Daniel Santos dos Santos
Universidade Regional Integrada do Alto Uruguai e das Missoes, Brasil
ORCID: https://orcid.org/0000-0002-6657-7924
Secretaria Municipal de Cacequi, Brasil
E-mail: danielsantosdoss@ hotmail.com
Rafaella França Torres
ORCID: https://orcid.org/0000-0002-9291-5341
Hospital São Roque, Brasil
E-mail: rafaellaftorres@ hotmail.com

\begin{abstract}
Resumo
O câncer de colo do útero (CCU) é considerado um problema para saúde pública em países em desenvolvimento manifestando altos índices de incidência de mortalidade decorrentes dessa patologia entre mulheres de nível socioeconômico baixo e em diversos períodos reprodutivos de suas vidas. O presente artigo objetiva obter resultados de dados elaborados através de pesquisas já publicadas sobre o assunto, no qual os pesquisadores são ferramentachave na compreensão e explicação da questão-problema. Para a efetivação do artigo, foi realizado uma revisão bibliográfica do tipo revisão integrativa de literatura- a busca aconteceu durante o mês de maio de 2021. Para a seleção dos estudos foi realizada uma busca online em periódicos e nas bases de dados: Scientific Electronic Library Online (SciELO), Ministério da Saúde e Instituto Nacional de Câncer (INCA). A busca dos materiais resultaram na seleção de 22 artigos, e após leitura criteriosa foram selecionados e utilizados nesta pesquisa, 15 artigos os quais atendiam a proposta do estudo. É indispensável que os enfermeiros prezem e sejam engajados na evolução do atendimento, para que haja mudança nos modelos mecanicistas, que focam apenas na doença, e passem a defender e realizar o atendimento de forma humanizada, acolhedora, empática, responsável e resolutiva. Valorizar a mulher em sua totalidade, considerar e compreender suas experiências, buscar uma troca de conhecimentos tornando o ambiente confortável e realizar as orientações de enfermagem o mais próximo possível da realidade em que a usuária está inserida, para que assim haja maior probabilidade seguimento e resolução.
\end{abstract}

Palavras-chave: Câncer de colo do útero; Enfermagem; Prevenção.

\begin{abstract}
Cervical cancer (CCU) is considered a public health problem in developing countries, showing high rates of mortality from this pathology among women of low socioeconomic status and in different reproductive periods of their lives. This article aims to obtain results from data elaborated through research already published on the subject, in which researchers are a key tool in understanding and explaining the problem-question. To carry out the article, a bibliographic review of the integrative literature review type was carried out - the search took place during the month of May 2021. For the selection of studies, an online search was performed in journals and in the following databases: Scientific Electronic Library Online (SciELO), Ministry of Health and National Cancer Institute (INCA). The search for materials resulted in the selection of 22 articles, and after careful reading, 15 articles were selected and used in this research, which met the study proposal. It is essential that nurses appreciate and be engaged in the evolution of care, so that there is a change in mechanistic models, which focus only on the disease, and start to defend and provide care
\end{abstract}


in a humanized, welcoming, empathetic, responsible and resolute manner. Valuing the woman as a whole, considering and understanding her experiences, seeking an exchange of knowledge making the environment comfortable and carrying out nursing guidelines as close as possible to the reality in which the user is inserted, so that there is greater likelihood of follow-up and resolution.

Keywords: Cervical cancer; Nursing; Prevention.

\section{Resumen}

El cáncer de cuello uterino (UCC) es considerado un problema de salud pública en los países en desarrollo, presentando altas tasas de mortalidad por esta patología entre mujeres de bajo nivel socioeconómico y en diferentes períodos reproductivos de su vida. Este artículo tiene como objetivo obtener resultados a partir de datos elaborados a través de investigaciones ya publicadas sobre el tema, en las que los investigadores son una herramienta clave para comprender y explicar el problema-pregunta. Para la realización del artículo se realizó una revisión bibliográfica del tipo revisión integradora de la literatura, la búsqueda se realizó durante el mes de mayo de 2021. Para la selección de estudios se realizó una búsqueda en línea en revistas y en las siguientes bases de datos: Scientific Electronic Library Online (SciELO), Ministerio de Salud e Instituto Nacional del Cáncer (INCA). La búsqueda de materiales resultó en la selección de 22 artículos, y luego de una lectura atenta, se seleccionaron y utilizaron 15 artículos en esta investigación, que cumplieron con la propuesta de estudio. Es fundamental que el enfermero aprecie y se involucre en la evolución del cuidado, para que se produzca un cambio en los modelos mecanicistas, que se enfocan solo en la enfermedad, y comience a defender y brindar el cuidado de una manera humanizada, acogedora, empática, responsable y decidida. camino. Valorar a la mujer como un todo, considerando y comprendiendo sus vivencias, buscando un intercambio de conocimientos que facilite el ambiente y llevando a cabo las pautas de enfermería lo más cerca posible de la realidad en la que se inserta la usuaria, de modo que haya mayor probabilidad de seguimiento. arriba y resolución.

Palabras clave: Cáncer de cuello uterino; Enfermería; Prevención.

\section{Introdução}

O câncer de colo do útero (CCU) é considerado um problema para saúde pública em países em desenvolvimento manifestando altos índices de incidência de mortalidade decorrentes dessa patologia entre mulheres de nível socioeconômico baixo e em diversos períodos reprodutivos de suas vidas (Rocha et al., 2020).

O CCU, também é chamado de câncer cervical, é provocado pela infecção resistente por algumas espécies do Papilomavírus Humano (HPV) (chamados de tipos oncogênicos). A infecção genital por esse vírus é bastante recorrente e não causa, na maioria das vezes doença. Porém, em algumas situações, acontecem modificações celulares que podem progredir para o câncer. Essas modificações são encontradas facilmente no exame preventivo (também conhecido como Papanicolaou ou Papanicolau), e possuem em quase a totalidade dos casos, cura. Por isso, é de extrema relevância a realização periódica desse exame (INCA, 2021).

Este tipo de câncer é descrito pela alteração descontrolada do epitélio de revestimento do órgão, e compromete o tecido subjacente, podendo alastrar-se para estruturas e órgãos próximos (Aoyama et al., 2019).

Existem dois principais tipos de carcinomas invasores do colo do útero, os quais dependem da origem do epitélio acometido: O carcinoma, epidermoide, tipo mais incidente e que compromete o epitélio escamoso (equivale a cerca de $80 \%$ dos casos) e o adenocarcinoma, tipo mais esporádico e que compromete o epitélio glandular em 10\% dos casos (INCA, 2021).

O CCU se caracteriza como o terceiro câncer mais comum no mundo, consistindo a quarta causa de morte por câncer entre as mulheres, categorizando-se como o segundo câncer mais recorrente no sexo feminino. Essas altas taxas indicam as falhas no rastreamento e na detecção precoce de lesões pioneiras deste tipo de neoplasia, o que procede um diagnóstico em estágio avançado da doença e, como consequência, pior sobrevida (INCA, 2021).

Levando em consideração que no contexto de Atenção Básica à Saúde (APS), porta de entrada para o Sistema único de saúde, e onde a coleta do material citopatológico é realizada com frequência, sendo esse exame uma atividade preventiva é uma atribuição privativa do enfermeiro (Cofen, 2015), compreendemos que seu papel é primordial, pois o mesmo possui contato direto com a população atendida no âmbito da APS (Ministério da saúde, 2013). 
É importante destacar a relevância desta resolução, considerando que a sua efetivação pode favorecer para o atendimento mais qualificado às usuárias, e inclusive para aprimorar demarcação do espaço profissional dos enfermeiros. É relevante haver empoderamento por parte dos enfermeiros para que executem a sua profissão com compromisso, responsabilidade e qualidade, primando sempre pelos preceitos éticos estabelecidos na lei de exercício profissional, com o propósito de garantir melhorias na assistência aos usuários (Oliveira et al., 2020).

Neste sentido, é preciso que este profissional desenvolva seu papel de educador, propagando seu conhecimento e pesquisando atividades e estratégias que desperte interesse e chamem atenção da população feminina, tendo em vista que são o público alvo deste obstáculo, para as ações de prevenção, orientando, encorajando e cooperando no que for preciso para que se construa uma relação de confiança, o que possibilitará a promoção da saúde e prevenção da doença, e se for necessário, o acompanhamento do tratamento (Avila et al., 2016).

\section{Metodologia}

O artigo utilizou-se do método de revisão narrativa da literatura, cujo objetivo é a reunião e síntese de resultados sobre o tema, apresentando e potencializando a construção de conhecimentos para possibilitar a incorporação de evidências na prática clínica. A revisão narrativa diferencia-se das demais revisões por permitir a inclusão de pesquisas de métodos diversos, proporcionado conhecimento mais amplo da literatura para conhecer e entender o tema estudado (Medeiros et al., 2015).

$\mathrm{O}$ artigo objetiva obter resultados de dados elaborados através de pesquisas já publicadas sobre o assunto, no qual os pesquisadores são ferramenta-chave na compreensão e explicação da questão-problema. Para a efetivação do artigo, foi realizado uma revisão bibliográfica do tipo revisão integrativa de literatura- a busca aconteceu durante o mês de maio de 2021 - foram incluídas as fontes de artigos on-line.

Para a seleção dos estudos foi realizada uma busca online em periódicos e nas bases de dados: Scientific Electronic Library Online (SciELO), Ministério da Saúde e Instituto Nacional de Câncer (INCA).

Posteriormente, na segunda fase da metodologia, foram selecionadas todas as bibliografias consideradas importantes que estavam associadas ao objetivo apresentado. Como critérios de inclusão estabeleceram-se artigos com texto completo artigos no idioma português, publicados no período de 2015 a 2021 (o intuito de reunir estudos mais recentes). Foram usados como palavras-chave os seguintes descritores: câncer de colo do útero, enfermagem e prevenção.

Utilizou-se como critérios de exclusão: a inacessibilidade do texto completo por via eletrônica; estudos escritos em outras línguas; e trabalhos que não contemplavam a assistência de enfermagem na prevenção e promoção de saúde para população feminina no que diz respeito à realização do exame Papanicolau. Os artigos repetidos nas diferentes bases de dados foram considerados apenas uma vez. O tema pesquisado foi classificadoconforme os critérios de inclusão, viabilizando construção dos materiais posteriormente.

De acordo com a Resolução 466/2012, para o desenvolvimento do estudo não foi necessário o encaminhamento para o Comitê de Ética, pois o presente estudo não envolveu seres humanos participando da pesquisa para a coleta de dados (Brasil, 2012).

Para realização desta revisão, foram aplicadas as seguintes etapas: busca, avaliação crítica e a síntese das evidências disponíveis do tema investigado. Após as informações foram sintetizadas, agrupadas, categorizadas e analisadas a partir da análise de conteúdo de Minayo que compreendem: a pré-análise, a exploração do material, o tratamento dos resultados obtidos e a interpretação (Minayo, 2010). 


\section{Resultados e Discussão}

A busca dos materiais resultou na seleção de 22 artigos, e após leitura criteriosa foram selecionados e utilizados nesta pesquisa, 15 artigos os quais atendiam a proposta do estudo. Elencou-se os seguintes temas para desenvolvimento de referencial teórico: Fatores associados a não realização do exame Papanicolau por parte da população feminina; Estratégias de Prevenção e Diagnóstico Precoce; e Consulta de Enfermagem na Prevenção de Câncer Uterino.

\section{Fatores associados a não realização do exame Papanicolau por parte da população feminina}

Em relação aos aspectos psicológicos Rodrigues, Schönholzer e Lemes (2016) evidenciaram que as mulheres foram interrogadas sobre as razões que levam a rejeição em fazer o exame preventivo. Entre os sentimentos alegados na pesquisa, destacam-se três: medo (40\%), desconforto (30\%) e vergonha (30\%). Nesse sentido, reforça-se aqui a influência da prática do acolhimento pelo enfermeiro. O profissional precisa oportunizar um ambiente confortável e natural, explicar para a mulher o procedimento e buscar explanar corretamente as dúvidas da mesma antes da realização do exame.

Ainda que haja atualmente, diversas fontes de informações e transmissão de notícias, encontramos mulheres que não conhecem o exame preventivo e não sabem da sua relevância para a saúde feminina, especialmente nas classes sociais mais vulneráveis. Além do constrangimento que o exame ocasiona em muitas dessas mulheres, o receio e o medo do resultado do exame é fator de tamanha importância para a não procura deste. O Ministério da Saúde observou que o desconhecimento da mulher sobre o câncer, o nível baixo de escolaridade, a escassez de conhecimentos sobre o próprio corpo, a vergonha e o medo de fazer o exame são alguns dos obstáculos para não realizar (Brasil, 2017).

Além disso, as pesquisas ressaltam a respeito da importância das orientações sobre a prevenção do HPV, que é transmitido através do contato íntimo, e que o controle das Infecções Sexualmente Transmissíveis (ISTs) reduz de maneira significativamente o risco da mulher desenvolver o CCU, além da magnitude de fazer o exame Papanicolau para prevenção ou detecção precoce deste tipo de câncer (Brasil, 2018).

Os inúmeros obstáculos enfrentados atualmente pelas mulheres para a realização do Papanicolau estão envolvidos diretamente com a carência de informações ofertadas pelos profissionais de saúde, e grande parte das vezes as usuárias desconhecem o papel do enfermeiro como educador (Lima et al., 2016) Logo, é de extrema importância que os profissionais enfermeiros reconheçam suas atribuições e desempenhem suas funções com engajamento e responsabilidade, para que assim seja possível alcançar a valorização necessária para esse profissional que possui um papel indispensável para saúde da população.

Foi possível constatar que a desinformação e a dificuldade de marcação do exame são os fatores prevalentes para não realização. A carência da atuação na educação em saúde e a busca ativa da população feminina na comunidade foram itens dignos de moldagem nas estratégias de atuação, por parte dos profissionais de saúde (Barbosa et al., 2017). Nesse sentido, é fundamental a organização dos atendimentos nos serviços de saúde, bem como acolhimento adequado, escuta qualificada e assistência que atendam as necessidades das usuárias que ali buscam atendimento. Essa organização, também é necessária para realizar a busca ativa de maneira adequada, inserindo as usuárias nos serviços e melhorando a qualidade de vida dessa população.

Apesar de todos os avanços do mundo moderno, o conhecimento das mulheres sobre o a coleta do Papanicolau, ainda é baixo a adesão das mesmas ao exame, e inúmeros fatores fazem com que elas o desistam de realizar o mesmo, o que acaba ocasionado uma menor chance de cura, se o diagnóstico for positivo (Dantas, et al., 2018). Neste momento, é fundamental que haja por parte do profissional um olhar empático, o qual busque tornar o ambiente o mais acolhedor e tranquilo possível, para que todo o atendimento se suceda tranquilamente e o procedimento de coleta do Papanicolau seja o mais natural e menos traumático possível, para que assim haja a criação de um vínculo de confiança e a usuária sinta-se confortável para todos os 
retornos necessários e também para expor suas dúvidas e receios a respeito de sua saúde para o profissional, neste momento, o enfermeiro faz saúde e proporciona qualidade de vida de maneira eficaz.

Foram evidenciadas diversas motivações para a busca da realização dos exames preventivos, tais como: a iniciação sexual, a situação obstétrica, a existência de sinais e sintomas ginecológicos, e o estimulo dos familiares e de profissionais da saúde, fortalecendo a importância da busca ativa e do elo entre esses profissionais e a população. O constrangimento para expor o corpo, o a vergonha, o medo em sentir dor e até mesmo a espera pelo resultado do exame aumentam a vulnerabilidade das mulheres à essa patologia (Acosta et al., 2017). Dessa maneira, comprova-se mais uma vez que o acolhimento, a escuta qualificada e o ambiente confortável por parte do enfermeiro são pontos significativos para que a mulher sinta-se mais tranquila e veja o atendimento como um momento de prevenção de saúde e melhora da sua própria qualidade vida.

Mesmo que o rastreamento do CCU seja indispensável para intervenções em tempo oportuno, importante parcela das mulheres ainda não adota a realização do exame por mitos e tabus, crenças e atitudes em saúde, bem como organização do serviço. Nesta perspectiva, precisa haver uma interação de maneira mais efetiva por parte dos profissionais de saúde para com a usuária, através do recuperação da equidade no cuidado que realiza, a individualização da assistência e a criação do vínculo de segurança e confiança que se sobreponha a vergonha, ao medo, dificuldades de acesso e à prática do autocuidado responsável (Silva et al., 2015).

Dentre tantas aptidões que são necessárias que o profissional enfermeiro possua, estar apto a trabalhar psicologicamente e emocionalmente às mulheres é mais uma delas, que pode passar despercebido, mas as mesmas tendem, na maioria das vezes a apresentaram inúmeros sentimentos negativos, e estes são considerados importantes fatores para a desmotivação das mulheres ao realizarem a prevenção do câncer de colo uterino (Amaral, Gonçalves \& Silveira 2017).

\section{Estratégias de Prevenção e Diagnóstico Precoce}

Ainda existe muito o que se fazer em prol da saúde, é preciso que haja mais empenho por parte dos profissionais, devem ser mais humanizados e comprometidos com a mudança. O Governo precisa dar maior suporte a ESF junto com a participação da população. O enfermeiro é a principal peça para a prevenção, diagnóstico e tratamento, e deve atuar junto com todos esses citados, organizando uma estratégia para obter resultados positivos ao trabalhar em conjunto com sua equipe, envolvendo-os nos trabalhos de prevenção e promoção de saúde (Santos et al., 2020).

O exame Papanicolau efetuado pelo enfermeiro no âmbito da APS, possui importância em todo o contexto da saúde da mulher, tanto na prevenção como na identificação precoce de alterações e início do tratamento em tempo oportuno, com informações pertinentes sobre o estado clínico e os diagnósticos. Comprovou-se que a melhor maneira prevenção do CCU é a realização do exame Papanicolau e a observação precoce de qualquer anomalia, somado as orientações da equipe multidisciplinar com objetivo de explanar qualquer dúvida quanto aos exames e seus resultados e minimizar as preocupações sobre os mesmos (Maciel, Aoyama \& Souza 2020).

É indispensável acontecer dessa maneira um exercício constante de informações por parte dos profissionais de enfermagem por meio da educação permanente, pois, a carência de qualificação específica pode ocasionar dificuldades na obtenção de uma assistência de qualidade ofertada para os usuários em geral, pois é necessário que o enfermeiro esteja qualificado e atualizado sobre as temáticas necessárias (Amaral, Gonçalves \& Silveira 2017).

É necessário reforçar a prática das ações educativas empregadas na rotina de todos os atendimentos focados na população feminina, e ao mesmo tempo propagar as informações sobre quais são os fatores de risco no desenvolvimento desta neoplasia e a relevância da realização periódica do exame preventivo. Desta maneira, será possível minimizar a taxa de ocorrência dessa patologia (Lopes et al., 2019). 
Evidenciou-se também que para o desempenho apropriado das ações do enfermeiro é indispensável a implantação e continuidade da política de educação permanente, pois a atuação profissional do enfermeiro que desempenha estratégias na prevenção do CCU é um processo permanente, responsável e dinâmico, o qual vai para além da graduação, e se estende durante toda sua carreira profissional (Santana et al., 2020). São necessárias estratégias de atualização, valorização e motivação dos profissionais, para estejam sempre empenhados e engajados em prevenir e promover saúde para a população feminina.

Mesmo que haja as políticas de saúde em nosso país voltadas à saúde da mulher, as quais compreendem campanhas de sensibilização e disseminação da importância da realização do exame preventivo, ainda observa-se que a população feminina é uma categoria consideravelmente vulnerável às ISTs e ao CCU. Por essa razão, há a indispensabilidade de investimentos maiores para o elaboração de ações de promoção à saúde, estratégias essas que sejam eficientes para modificar esse cenário, essencialmente no que refere-se à disponibilização de suporte educacional em saúde, provendo informações de maneira direita e adequada, oportunizando esclarecer mitos e desmistificar tabus sobre esse tipo de câncer (Santana et al., 2020).

Evidenciou-se que a relevância do enfermeiro em realizar ações educativas com as usuárias da comunidade, para que seja possível transmitir o máximo de informações, conscientizando não só a si como também a seus parceiros, pois o câncer de colo do útero quando detectado precocemente tem $100 \%$ de cura (Amaral, Gonçalves \& Silveira 2017). As ações educativas, podem ser realizadas de diversas formas, considera-se importante proporcionar momentos de troca de conhecimentos e experiências entre o profissional enfermeiro, as usuárias e seus acompanhantes, para que assim haja valorização dessas mulheres, maior alcance na participação de todos e consequentemente nas informações transmitidas.

Devido a equipe de enfermagem manter uma relação mais próxima à população e de dispor de uma formação mais generalista com focados na humanização e educação em saúde, podem colaborar de forma importante para o enfrentamento do CCU quando este é diagnosticado. Sendo, consequentemente, de valor extremo que o enfermeiro reconheça o as função e assuma as suas responsabilidades perante a esse contexto, em busca, dessa maneira, desempenhar estratégias que atinjam e sensibilizem a população feminina de forma geral e reforce a temática de prevenção (Santana et al., 2020).

Dessa forma, é preciso haver uma busca ativa continuada das usuárias que realizam os exames, porém não voltam para buscar o resultado do mesmo, pois apenas a realização do exame não é suficiente para que se dê continuidade à prevenção do câncer de colo do útero, visto que sem o retorno não é possível informar sobre a necessidade ou não de tratamento medicamentoso, orientações e encaminhamentos necessários (Amaral, Gonçalves \& Silveira 2017).

Ao tratar-se de estratégias para educação em saúde, o integrante da equipe multidisciplinar, profissional enfermeiro, precisa engajar-se e esforçar-se com objetivo de promover mudanças, na concepção feminina, a respeito do exame preventivo e suceder ações para que mais mulheres façam o exame, como rodas de conversas, encontros em locais próprios, empresas, comércio, entre outros (Lopes et al., 2019).

Além do mais, o enfermeiro tem de continuar com sua atuação engajada nas orientações de adesão e continuidade ao exame preventivo, bem como à disseminação de informações a respeito das maneiras de prevenção desse câncer, uma vez que ações comportamentais têm potencial para reduzir os riscos a que as usuárias estão expostas. Orientações quanto aos métodos diagnósticos, tratamento, efeitos adversos de cada terapia, cuidados essenciais e necessidade de apoio familiar são fundamentais para uma assistência de qualidade (Lopes et al., 2019).

A enfermagem trabalha de modo direto na realização do exame do Papanicolau e assim é indispensável que haja a propagação das informações necessárias; não somente desse exame, o qual é bem temido, mas também para todos os outros, focando na saúde de maneira geral. Pois é notório que essas ações permitem dar prioridade para a promoção à saúde e a prevenção de doenças. Vale ressaltar então, que o uso de tecnologias educativas colabora para o aprendizado da população de maneira simplificada, bem como auxilia o profissional no repasse de informações (Lima et al., 2019). 
$\mathrm{O}$ rastreamento do $\mathrm{CCU}$, fortemente relacionado a feminilidade, deve oportunizar possibilidades para as mulheres conquistarem autonomia a respeito dos seus corpos e sua saúde, levando-as a afastar-se da postura de passividade a que é submetida na expressão de sua sexualidade. A prevenção desta neoplasia precisa contemplar as ações cujo objetivo seja oportunizar a passagem da mero consentimento para uma "participação informada", por parte das usuárias, no rastreamento, fundamentada não somente na propagação de orientações, mas, acima de tudo, no reconhecimento dos seus valores, experiências e práticas (Souza \& Costa 2015).

\section{Consulta de enfermagem na prevenção de câncer uterino}

Machado e Andres (2021) compreenderam que a maneira mais eficaz de alcançar maior confiança por parte dos usuários durante as consultas de enfermagem, é usá-la cada vez mais como metodologia de trabalho, introduzindo-a nos programas de saúde e valorizando-a como uma estratégia com grande capacidade para produzir um atendimento cada vez mais efetivo, responsável e humanizado à população, aliando o embasamento teórico ao prático em constantes atualizações.

O CCU é uma das temáticas mais referidas atualmente, dentro de um contexto geral, isso se deve ao fato de que é uma das neoplasias que mais atinge as mulheres brasileiras, junto com o câncer de mama. Apesar do Governo elaborar diversos programas, não soluciona todas as dificuldades da população, levando em consideração que mesmo depois da implantação dos programas a incidência deste tipo de câncer continuou elevada. Sendo assim, a atuação da Equipe de Saúde da Família e principalmente o enfermeiro possuem um papel essencial dentro deste contexto (Santos et al., 2020).

Comprovou-se que o profissional enfermeiro é o principal responsável dentro da APS pela prevenção e promoção da saúde. Desta forma, pela proximidade com as usuárias é capaz de observar os obstáculos encontrados para a realização do exame citopatológico, podendo nesse sentido, buscar alternativas adequadas que solucionem os mesmos, por meio de uma postura crítico-reflexiva para a realização de uma assistência humanizada e responsável (Amaral, Gonçalves \& Silveira 2017).

O enfermeiro possui um papel preponderante, tanto na prevenção deste tipo de câncer, quanto na promoção e prevenção da saúde da mulher em todas as áreas. O diagnóstico precoce e o uso de tecnologia simplificada proporcionam um tratamento eficaz e efetivo, pois este apresenta etapas bem estabelecidas, de maneira mais rápida e prática. Outra comprovação proporcionada por este estudo, trata-se da proximidade da relação do enfermeiro com as usuárias e que esse vínculo humanizado só tem a auxiliar para o sucesso da realização dos exames preventivos (Maciel, Aoyama \& Souza 2020).

Constata-se que as mudanças no estilo de vida das mulheres e a efetivação de um acolhimento adequado por parte do profissional enfermeiro durante as consultas de rotina são fatores essenciais e preventivos encontradas na literatura. Notou-se, também, que é atribuição do profissional de enfermagem e da equipe multidisciplinar incluir as mulheres nas ações realizadas pela APS a fim de acolher, orientar, e prevenir doenças e promover saúde, e com isso oportunizar uma melhora na qualidade de vida das mesmas (Santana et al., 2020).

O profissional de saúde, em especial o enfermeiro, dispõe de um primordial papel no contexto da prevenção do CCU é atribuição deste, planejar e desenvolver intervenções que proporcionem a integralidade e equidade, que envolvam a saúde da mulher (Tavares et al., 2017). Torna-se fundamental que o enfermeiro, além de chamar atenção e buscar a conscientização das mulheres não somente para realização do exame, mas também a adesão de práticas saudáveis no seu estilo de vida, para promover saúde e melhorar a qualidade de vida em todos os aspectos.

É indispensável que o enfermeiro trabalhe de maneira ética e responsável, para respeitar e compreender sem julgamentos os estigmas associados aos valores morais, religiosos e culturais, dando espaço para um olhar holístico e responder questões de gênero que possuem impacto na sexualidade, na saúde e na adesão ao exame citopatológico. É preciso também, que os gestores e profissionais de saúde saibam bem o seu papel e desempenhem com seriedade, responsabilidade e sensibilidade durante cada etapa das estratégias e ações voltadas para o controle do CCU (Lopes et al., 2019). 
Também é indispensável por parte do profissional, o preenchimento adequado de todos os dados de cada paciente. Que o enfermeiro mantenha qualidade na hora da realização do exame, com coletas de materiais suficientes para que não seja necessário se repetir o exame, o que pode causar um desconforto maior ainda na mulher, como também medo e ansiedade pelo resultado (Lopes et al., 2019).

Percebe-se a necessidade e a importância de uma atuação mais ativa do enfermeiro em explanar informações corretas e atualizadas sobre o $\mathrm{CCU}$, o exame preventivo, e a relevância de sua realização, bem como as consequências da não periodização do exame. Deixando de lado a atuação de maneira mecanicista como o modelo biomédico que só foca na cura, mas atuando de maneira mais acolhedora, humana e empática, para que seja possível possuir a confiança das mulheres e sensibilizarem a tomar decisões para melhorar a qualidade de vida e com isso prevenir e promover saúde (Barbosa et al., 2019).

A realização do exame do Papanicolau é conceituada como o principal procedimento de prevenção do CCU sendo o enfermeiro habilitado e instruído para fazer a coleta no qual é necessário assegurar a organização, a integralidade e a qualidade do programa de prevenção e rastreamento do CCU (Da Costa et al., 2017).

Da Costa et al (2017) apontam razões que influenciam na prevenção e rastreamento precoce da neoplasia, como a desorganização do serviço prestado, carência de humanização no decorrer do atendimento, insegurança das mulheres em relação ao exame, situação econômica e sociocultural e escassez de conhecimento das mulheres sobre as DSTs. Esses fatores contribuem negativamente para que a mulher não procure a assistência preventiva, o que necessita por parte do serviço de saúde uma busca ativa, quando infelizmente, as alterações já estão em uma fase mais avançada, o que torna o atendimento curativo e não preventivo.

Tanto a consulta de enfermagem como o exame citopatológico não precisam seguir somente os procedimentos rotineiros da realização deste momento por meio de um modelo sindrômico, o enfermeiro deve dar prioridade para um diálogo no qual esteja aberto para ouvir a usuária e se agir com empatia. É imprescindível que o enfermeiro possua uma visão holística, praticando o seu trabalho de maneira humanizada e integral (Amaral, Gonçalves \& Silveira 2017).

O CCU é das patologias crônico-degenerativas que possui elevado grau de letalidade morbidade, mas da mesma forma que apresenta considerável probabilidade de cura quando diagnosticado precocemente (Rodrigues, Schönholzer \&Lemes 2016). Neste sentido, comprova-se a dimensão da atuação do profissional enfermeiro na detecção precoce dos sinais de alteração, na prevenção desta patologia e na promoção de saúde, melhorando consideravelmente as chances de cura e qualidade de vida dessa população.

O enfermeiro precisa transmitir o máximo de segurança possível para a usuária em atendimento, para que esta possa compreender a realização do exame, sentir-se segura e preservada e ao mesmo tempo entender a importância da realização do exame (Amaral, Gonçalves \& Silveira 2017). Nesse sentido, a consulta de enfermagem torna-se um momento indispensável para explicar a importância da prevenção e detecção precoce dos sinais de alteração, promovendo saúde e melhorando a qualidade de vida das usuárias em que ali são atendidas.

\section{Considerações Finais}

É indispensável que os enfermeiros prezem e sejam engajados na evolução do atendimento, para que haja mudança nos modelos mecanicistas, que focam apenas na doença, e passem a defender e realizar o atendimento de forma humanizada, acolhedora, empática, responsável e resolutiva. Valorizar a mulher em sua totalidade, considerar e compreender suas experiências, buscar uma troca de conhecimentos tornando o ambiente confortável e realizar as orientações de enfermagem o mais próximo possível da realidade em que a usuária está inserida, para que assim haja maior probabilidade seguimento e resolução. 
Diante dos resultados obtidos e expostos por meio deste estudo, comprovou-se que há uma lacuna importante na disseminação e principalmente no alcance de informações e orientações apropriadas para a população feminina a respeito dos cuidados necessários com sua própria saúde, especialmente na questão da realização do exame Papanicolau pra prevenir e promover saúde e minimizar agravos.

Comprovou-se ainda, de maneira unanime após a leitura dos artigos selecionados, que o profissional enfermeiro é uma peça transformadora e indispensável no que diz respeito a assistência prestada para as mulheres. Através desse profissional, é possível planejar e desenvolver ações de educação em saúde e durante as consultas de enfermagem é possível ouvir, compreender, acolher e orientar as mulheres de maneira individual e empática, alcançando dessa forma maior efetividade nas ações profissionais.

Levando em consideração a relevância do tema e principalmente da atuação do profissional no contexto de prevenção e promoção da saúde da população feminina, sugere-se com esse estudo, que cada vez mais hajam mais pesquisas evidenciando a importância do engajamento profissional no que diz respeito as ações de educação em saúde e sensibilização das mulheres para o autocuidado.

\section{Referências}

Acosta, D. F. et al. (2017) Vivenciando o exame Papanicolau: entre o (não) querer e o fazer. Rev enferm UFPE online; 11 (8).

Amaral, M. S., Gonçalves, A. G. \& Silveira, L. C. G. (2017) Prevenção do câncer de colo de útero: a atuação do profissional enfermeiro nas unidades básicas de saúde. Revista Científica FacMais; (3) 1.

Aoyama, E. A. et al. (2019) Assistência de enfermagem na prevenção do câncer de colo do útero. Braz. J. Hea. Rev; (2) 1.

Avila, F. A. et al. (2016) A Importância do Exame Papanicolau na Prevenção e Manutenção da Saúde da Mulher. Sem. De Enfermagem Fevalle.

Barbosa, L. C. R. et al. (2017) Percepção de mulheres sobre os fatores associados a não realização do exame Papanicolau. Interfaces Científicas - Saúde e Ambiente; (5) 3 .

Brasil, Ministério da Saúde (BR) (2017). ABC do Câncer. Abordagem básica para o controle do câncer.

Brasil, Ministério da Saúde (BR) (2018). Secretaria Nacional de Assistência à Saúde. Instituto Nacional do Câncer - INCA.

Brasil. Ministério da Saúde. (2012) Conselho Nacional de Saúde. Resolução n ${ }^{\circ}$ 466, de 12 de dezembro de 2012 . Aprova as diretrizes e normas regulamentadoras de pesquisas envolvendo seres humanos.

Brasil. Ministério da Saúde. (2013) Secretaria de Atenção à Saúde. Departamento de Atenção Básica. Controle dos cânceres do colo do útero e da mama.

Cofen (2015). Parecer de conselheiro federal N 190/2015/COFEN.

Da Costa, F. K. M. et al. (2017) Os desafios do enfermeiro perante a prevenção do câncer do colo do útero. RGS; 17 (1).

Dantas, P. V. J, et al. (2018) Conhecimento das mulheres e fatores da não adesão acerca do exame Papanicolau. Rev enferm UFPE online; 12 (3).

Instituto Nacional do Câncer - INCA (2021). Conceito e Magnitude. .Instituto Nacional do Câncer - INCA (2021). Tipos de câncer. Câncer do colo do útero.

Instituto nacional do câncer - INCA. (2014) Ações de enfermagem para o controle do câncer: uma proposta de integração ensino-serviço. (3a ed.), Rev. atual. Ampl. -

Lima, H. F. et al. (2016) Saúde e educação popular com mulheres sobre o exame papanicolau: relato de experiência. Rev EEDIC.

Lopes, J. C. et al. (2019) O Papel do Enfermeiro no conhecimento das Mulheres acerca do Exame de Papanicolau. Rev. Mult. Psic; (13) 47.

Machado, L. B. \& Andres, S. C (2021) A consulta de enfermagem no contexto da Atenção Primaria em Saúde: Relato de experiência. Rev Research, Society and Development; (10) 1 .

Machado, L. F. \& Peixoto, M. (2016) A Importância do Exame Papanicolau na Prevenção e Manutenção da Saúde da Mulher. Sem. De Enfermagem Fevalle.

Maciel, L. A. M., Aoyama, E.A. \& Souza, R. A. G. (2020) A importância do exame papanicolau realizado pelo enfermeiro para o diagnóstico do câncer no colo uterino. ReBIS; 2 (2).

Medeiros, R. K. S et al. (2015) Modelo de validação de conteúdo de Pasquali nas pesquisas em Enfermagem. Rev Enferm Ref; (4) p 127-35.

Minayo, M. C. S. (2010). O desafio do conhecimento: pesquisa qualitativa em saúde. (12. ed.), Hucitec 
Research, Society and Development, v. 10, n. 7, e30910716648, 2021

(CC BY 4.0) | ISSN 2525-3409 | DOI: http://dx.doi.org/10.33448/rsd-v10i7.16648

Oliveira, L. L. et al. (2020) Exclusividade na coleta de material para exame de colpocitologia oncótica: percepção dos enfermeiros. REUFSM; (10).

Rocha, M. D. H. et al. (2020) Prevenção do câncer de colo de útero na consulta de enfermagem para além do Papanicolau; Revista Cereus; (12) 1.

Rodrigues, J. Z., Schönholzer, T. E. \& Lemes, A. G. (2016). Perfil das mulheres que realizam o exame Papanicolau em uma Estratégia de Saúde da Família. $J$ Nurs Health; 6 (3).

Santana, A. R. S. et al. (2020) A atuação do enfermeiro na estratégia de saúde da família para prevenção do câncer de colo uterino. ReBIS; 2 (3).

Santos, J. D. F. et al. (2020) O papel do enfermeiro na prevenção do câncer do colo uterino. ReBIS; 2 (1).

Silva, M. A. S et al. (2015) Fatores relacionados a não adesão à realização do exame de Papanicolau. Rev Rene; 16 (4).

Souza, A. F. \& Costa, L. H. R. (2015) Conhecimento de Mulheres sobre HPV e Câncer do Colo do Útero após Consulta de Enfermagem. Revista Brasileira de Cancerologia; 61 (4).

Tavares, N. C. M. et al. (2017) Perfil clinico, sexual e reprodutivo das mulheres que realizaram o exame papanicolau no ambulatório de uma faculdade em São Luís-MA. R. Interd; (10) 1 